\title{
A NOTE ON TRIANGULAR OPERATORS ON SMOOTH SEQUENCE SPACES
}

\section{ELIF UYANIK AND MURAT HAYRETTIN YURDAKUL}

Abstract. For a scalar sequence $\left(\theta_{n}\right)_{n \in \mathbb{N}}$, let $C$ be the matrix defined by $c_{n}^{k}=\theta_{n-k+1}$ if $n \geqslant k$, $c_{n}^{k}=0$ if $n<k$. The map between Köthe spaces $\lambda(A)$ and $\lambda(B)$ is called a Cauchy Product map if it is determined by the triangular matrix $C$. In this note we introduced some necessary and sufficient conditions for a Cauchy Product map on a nuclear Köthe space $\lambda(A)$ to nuclear $G_{1}$-space $\lambda(B)$ to be linear and continuous. Its transpose is also considered.

Mathematics subject classification (2010): 47B37, 46A45.

Keywords and phrases: Köthe spaces, smooth sequence spaces, Cauchy product.

\section{REFERENCES}

[1] L. CRone And W. Robinson, Diagonal maps and diameters in Köthe spaces, Israel J. of Math. 17, (1975), 13-22.

[2] G. KöTHE, Topological Vector Spaces 1, Springer-Verlag 1969.

[3] R. Meise And D. Vogt, Introduction to Functional Analysis, Clarendon Press, Oxford, 1997.

[4] A. Pietsch, Nuclear Locally Convex Spaces, Springer-Verlag, Berlin-New York, 1972.

[5] M. S. Ramanujan And T. TerzioĞLu, Subspaces of smooth sequence spaces, Studia Math. 65, (1979), 299-312.

[6] T. TerZioĞLu, Die diametrale Dimeansion von lokalkonvexen Räumen, Collect. Math. 20, (1969), 49-99. 